Frédérique CHEVILLOT, Anna NORRIS, dirs, Des femmes écrivent la guerre

Grignan, Éd. Complicités, coll. Entre l'art et la littérature, 2007, 237 p.

\title{
Catherine Gravet
}

\section{OpenEdition}

Journals

Édition électronique

URL : http://journals.openedition.org/questionsdecommunication/1891

DOI : 10.4000/questionsdecommunication.1891

ISSN : 2259-8901

Éditeur

Presses universitaires de Lorraine

Édition imprimée

Date de publication : 1 juillet 2008

Pagination : 355-359

ISBN : 978-2-86480-952-4

ISSN : 1633-5961

Référence électronique

Catherine Gravet, "Frédérique cheVILLot, Anna norRIs, dirs, Des femmes écrivent la guerre », Questions de communication [En ligne], 13 | 2008, mis en ligne le 01 juillet 2010, consulté le 08 avril 2021. URL: http://journals.openedition.org/questionsdecommunication/1891 ; DOI : https://doi.org/10.4000/ questionsdecommunication.1891

Ce document a été généré automatiquement le 8 avril 2021.

Tous droits réservés 


\section{Frédérique CHEVILLOT, Anna NORRIS, dirs, Des femmes écrivent la guerre}

Grignan, Éd. Complicités, coll. Entre l'art et la littérature, 2007, 237 p.

\section{Catherine Gravet}

\section{RÉFÉRENCE}

Frédérique CHEVILLOT, Anna NORRIS, dirs, Des femmes écrivent la guerre. Grignan, Éd.

Complicités, coll. Entre l'art et la littérature, 2007, 237 p.

1 L'écrivaine serbe en exil, Dubravka Ugresic, racontait, dans Le Monde diplomatique de septembre 2005, une scène d'un vieux film soviétique, Le Quarante et unième: en plein désert, une jeune et courageuse soldate de l'Armée rouge capture un ennemi, officier de la Garde blanche. Mais comme le papier à cigarettes vient à manquer et qu'elle est tombée amoureuse du séduisant prisonnier, la généreuse soldate offre les feuillets du carnet où elle a composé des poèmes. Pour Dubravka Ugresic, c'est un résumé métaphorique de l'histoire des lettres féminines : les femmes sont, au mieux, du côté du public, ou au service de l'écrivain ; elles étouffent leur créativité.

2 Pas de soldate-poète soviétique ici; l'essai collectif est dédié aux femmes et aux hommes qui refusent de faire la guerre même si Pamela Pears («Le mythe de la guerrière :Yamina Mechakra et LyThu Ho ", pp.125-137) envisage la "guerrière " comme une construction médiatique, d'ailleurs fort éloignée de la réalité, servant d'outil de propagande durant les guerres d'indépendance en Algérie et au Vietnam. Le titre du livre est explicite: les 16 contributions, dont 15 (il n'y a pas de notice biographique) rédigées par des femmes, francophones, sont consacrées à l'analyse de textes écrits par des femmes, en français, durant les guerres du xX siècle. En fonction de leurs expériences personnelles (majoritairement celles des deux guerres mondiales), elles apportent toutes un regard nouveau sur ce qu'elles ont vécu, parfois indirectement. Sur le thème choisi, se croisent donc deux paradigmes interprétatifs, études francophones et féministes, qu'il n'est plus besoin de justifier Frédérique 
Chevillot et Anna Norris, comme elles le signalent dans leur brève introduction, ont exclu les comparaisons avec les textes d'auteurs masculins, ainsi que les témoignages concentrationnaires. Est exclu aussi un éventuel parallèle avec le vécu de celles qui s'exprimeraient en russe, allemand, anglais.. Une belle symétrie partage en quatre sections (un peu arbitrairement: "Voix, femmes, guerres", p. 15; «Histoire(s) de guerre(s) », p. 77 ; «Des femmes, après la guerre... », p. 139 et "Elles écrivent leur guerre », p. 203), chaque fois quatre articles dont le plus court fait douze pages, le plus long vingt. On regrette l'absence d'index et de bibliographie générale, bien que, pour la seconde, une sélection des études des témoignages féminins ait été annoncée (p. 13).

Un peu moins de précipitation aurait peut-être permis d'ajouter quelques mots manquants, de corriger quelques coquilles, des erreurs de pagination, des maladresses typographiques, voire des problèmes de références, de-ci de-là. Ainsi la bibliographie de l'essai de Ruth Amossy («Les Récits des infirmières de 1914-1918», pp. 17-34), qui ouvre le recueil, est-elle lacunaire : il y manque quelques-uns des récits d'infirmières de 1914-18 (le sujet de la communication) ou des titres d'auteurs majeurs (à quels titres de Pierre Bourdieu, de Ruth Amossy elle-même est-il fait référence ?). Dommage, alors que la démonstration est magistrale. Rompue à l'art de l'argumentation, Ruth Amossy met au jour un corpus oublié de témoignages, pour la plupart conformistes, dont elle analyse avec rigueur les raisons pour lesquelles leurs auteures ne peuvent faire autrement que d'entrer dans la gangue de féminité stéréotypée où elles s'enferment. C'est que la femme salvatrice, consolatrice, soignante peut seule se faire entendre dans le chœur des patriotes de la Grande Guerre ; la lucidité subversive qui aurait dénoncé la violence guerrière aurait tout simplement été bâillonnée.

4 Nancy Sloan Goldberg (« Les Femmes, le civil et le soldats dans les romans de la Grande Guerre ", pp. 79-90) s'intéresse aussi, avec quelques pointes ironiques, à des textes oubliés, publiés durant la Première Guerre mondiale par des femmes, ici des professionnelles de l'écriture, qui assumèrent avec enthousiasme le rôle de propagandiste; en comparant des romans de femme à des romans d'homme (Le Feu d'Henri Barbusse comme parangon de misogynie), elle s'éloigne de la consigne des éditrices mais donne à voir une asymétrie intéressante : les femmes de lettres (Lucie Delarue-Mardrus, Colette Yver, Marcelle Tinayre, $\mathrm{M}^{\mathrm{me}}$ Yves Pascal, Camille Mayran.) doivent avant tout réfuter le "dalilaïsme" dont on accuse les femmes, traitresses rendues coupables de la défaite française. Toutes soulignent chez leurs protagonistes une transformation positive : la guerre en fait de véritables héroïnes, capables parfois de sortir de leurs rôles féminins traditionnels, d'assumer des responsabilités, de se sacrifier pour la patrie, de la reconstruire.

5 La littérature de jeunesse, souvent réservée aux femmes, n'est pas de celle qui laisse des traces indélébiles dans les histoires littéraires. Éléonore Hamaide (« La Seconde Guerre mondiale dans la littérature de jeunesse », pp. 187-203) voit dans la production de six auteures d'après-guerre une évolution intéressante. Dans les livres de Colette Vivier, Aranka Siegal, Yaël Hassan, Stéphanie Benson, Rachel Hausfater-Douïeb et Muriel Szac, la guerre, au départ extérieure aux protagonistes, permet la construction d'une identité qui plonge ses racines dans une histoire familiale, elle rapproche de ceux qui ont vécu cette tragédie et même des disparu-e-s, elle valorise les figures féminines.

6 La plupart des contributions s'attaquent à des auteures célèbres dans le champ littéraire francophone: Simone de Beauvoir Nina Bouraoui, Andrée Chedid, Colette, Assia Djebar Marguerite Duras, Agota Kristof Clara Malraux, Alice Rivaz, Marguerite 
Yourcenar... Ainsi la grande qualité de Colette (1873-1954), selon Josette Rico («Colette et la guerre : sur le front et de sa fenêtre. ", pp. 205218), est-elle de mettre la guerre à distance en n'évoquant, avec lyrisme, que ses conséquences sur la vie domestique de ses contemporaines. Un regard «à ras de terre ». Elle a pourtant vécu directement les deux guerres, que plusieurs critiques lui reprochent de ne pas avoir analysées. Ce parti pris de l'anecdote, du prosaïsme (qui va jusqu'aux conseils résignés pour survivre aux pénuries) n'exclut pas l'humour, dans ses billets sur la "mode des tranchées » par exemple, l'hommage vibrant à la vaillance des femmes, enfants, vieillards, et surtout la compassion émue pour les mutilés qui lui rappellent son père (le capitaine Jules Colette a perdu une jambe à la bataille de Melegnano en 1859).

7 La peur est souvent le sentiment majeur. Dans les nouvelles de Clara Malraux (lue par Colette Trout, «Clara Malraux : les Femmes et la Résistance au quotidien», pp. 35-48), elle domine les héroïnes au quotidien. Mais, alors que le virilisme propose à l'admiration des lecteur-rice-s des guerriers sans peur et sans reproche, elles résistent à leur manière et pour des raisons parfois troubles qui pourtant les subliment.

8 Valérie Baisnée ("Journal de guerre de Simone de Beauvoir», pp. 91-104) analyse les fonctions du Journal de Simone de Beauvoir en soulignant quelques caractéristiques inhérentes à l'écriture diariste en temps de guerre: suppléer à l'absence d'interlocuteurs (Sartre et Bost), comprendre plus intimement la guerre, retisser « le fil perdu du temps humain» et de l'histoire, trouver des références morales et philosophiques chez les écrivains de la Grande Guerre (mais Dabit est «emmerdant ", Green « minable », Gide " gâteux ».), combler ses angoisses, ébaucher des intrigues et se construire un style. La guerre, vécue comme rupture, favorise, ici aussi, l'émergence d'une femme, Simone de Beauvoir, plus consciente des réalités collectives, plus responsable.

9 Journal encore, ce que Marguerite Duras écrit en 1944-1946, dans l'angoisse, après l'arrestation de son mari résistant, Robert Antelme, mais qu'elle publiera 40 ans plus tard, remanié au point d'éveiller le soupçon d'imposture. Sandrine Rabosseau ("Marguerite Duras : la douleur d'écrire le crime de guerre », pp. 141-155) étudie les six textes qui constituent La Douleur en s'interrogeant sur les motivations de l'auteure et sur ses positions face au crime de guerre. Le devoir de vérité et de mémoire amène Marguerite Duras à fustiger les deux camps : bourreaux et victimes échangent leur rôles, chaque individu est capable de commettre des crimes, parfois au nom d'une idéologie, chaque être humain a sa part de responsabilité dans la Shoah. Marguerite Duras passe aux aveux: son sens de la justice même la pousse à torturer, son désir s'éveille pour le traitre séduisant, sa position dominante lui procure un plaisir quasi érotique, dérangeant. Malgré l'intransigeance de sa position durant l'épuration, son désarroi transparaît sans pathos ni effet de style.

Le terme "genre » utilisé par Nicolas di Méo à propos de Marguerite Yourcenar («Le genre à l'épreuve de la guerre chez Marguerite Yourcenar ", pp. 157-172) peut paraître ambigu quand on note les efforts obsessionnels de la romancière pour brouiller les pistes et tenter d'interdire au lecteur-rice l'identification qu'il-elle serait tenté-e de commettre entre auteure et narrateur-rice. Le masculin supplante souvent le féminin, même dans l'autobiographie, sans doute parce que Marguerite Yourcenar, s'inscrivant dans un courant néo-classique, estime que la légitimation passe par la dénégation obligatoire du féminin. Seul subsiste chez les héroïnes cet attribut, le don de soi, qui, 
loin de l'héroïsme guerrier que récuse la romancière, peut se muer en acte politique, en sacrifice, en vengeance.

11 L'absence des spécificités souvent reconnues à la littérature féminine a attiré l'attention de Catherine Bouthors-Paillart ( L'innommable en abyme: la trilogie d'Agota Kristof », pp. 233-244) sur Le Grand Cahier (1986), La Preuve (1988) et Le Troisième Mensonge (1991) de la Hongroise Agota Kristof: le "dénuement scripturaire», le bannissement de toute émotion ainsi que de toute prise de position morale sur les horreurs de la guerre en font un «monstre littéraire » mais constituent une mise en abyme particulièrement efficace de la « chose » innommable à dénoncer, dont on ne peut pourtant espérer témoigner.

Zupancic ("Chantal Chawaf : la déchirure originelle, le mal initial », pp. 219-232) décrit, elle, avec éloquence, l'écriture et le destin de Chantal Chawaf, alias Marie de la Montluel : née en 1943 d'une mère mourante, d'un père mort à cause de l'explosion d'une bombe sur le chemin de la maternité, elle est immédiatement adoptée. Tous les livres de Chantal Chawaf (du nom de son mari syrien), romans ou essais, sont autant d'entreprises thérapeutiques, pourtant impuissantes à guérir le traumatisme initial. La névrose consentie se transforme en force motrice. Les recherches dans les archives - les lois françaises de 1941 sur l'adoption ont permis l'effacement de ses origines -, le recours aux mythes - Perséphone, Déméter, Hécate -, la psychanalyse - Metka Zupancic renvoie à de nombreux essais sur les impacts psychiques de la guerre -, rien n'empêche l'auteure de considérer la déchéance du monde contemporain (pollution, nucléaire, politique sociale, matérialisme) comme une séquelle des guerres à dénoncer sans trêve.

De Viviane Forrester (née en 1925), journaliste, critique d'art et critique littéraire, essayiste, romancière, Anne-Marie Obatjek-Kirkwood ( Viviane Forrester : une guerre qui n'en finit pas ", pp.173-185) retient le recueil de nouvelles Ce soir, après la guerre (1992), et s'interroge sur les motivations d'une tentative autobiographique aussi tardive. 65-66 ans, n'est-ce pas pourtant un âge où, carrière parachevée, on se retrouve avec ses souvenirs et ses passions, peut-être, parfois, apaisées ? Pourquoi s'étonner de cette volonté de témoigner d'une tragédie, collective et individuelle, sans pareille dans l'histoire de l'humanité, inoubliable pour une adolescente juive ? Peut-on juger qu'il est trop tard pour exorciser l'horreur?

14 Si le "court $\mathrm{xx}^{\mathrm{e}}$ siècle » est celui des extrêmes dans la violence, la neutralité est paradoxalement vécue comme culpabilité par deux Suissesses, féministes et pacifistes, Alice Rivaz et Yvette Z'Graggen, comme le montre Joy Charnley (« Alice Rivaz et Yvette Z'Graggen : l'invivable neutralité helvétique », pp. 49-62). Il s'illustre aussi ailleurs que sur le continent européen, au Liban par exemple où Andrée Chedid (Debbie Mann, "Andrée Chedid: des actes de violence aux actes parole ", pp. 63-76) et ses héroïnes « transforme[nt] les actes de violence en actes de parole » et œuvrent ainsi pour la paix en faisant mentir l'étymologie masculine du mot « fraternité ».

Selon Milouda Louh («Figuration dans l'œuvre d'Assia Djebar», pp. 105124), Assia Djebar, militante pendant la guerre d'indépendance de l'Algérie, montre, du moins dans ses premiers romans, des personnages féminins, guerrières ou prostituées, dans une intimité comme éloignée de la réalité de la guerre. Ces femmes nouvelles, rendues visibles par la guerre, à qui l'on impose la claustration, ont pourtant un corps et des yeux qui leur permettent de connaître un érotisme inattendu. Depuis 1995, Assia Djebar souligne, en historienne, la continuité entre les bourreaux d'hier et ceux d'aujourd'hui, dénonce la violence et l'oubli, tout en s'engageant dans une recherche esthétique qui 
fait de L'Amour, la fantasia son œuvre la plus expérimentale. Mêlant histoire collective et histoire personnelle, recherche filmique et documentaire, "par l'entremise de la langue des assassins-géôliers ", l'écriture se fait "écrin du dit de femme", à la fois recréation et champ de bataille. C'est encore la guerre d'Algérie que Martine Fernandes, se heurtant à de multiples écueils théoriques, piste chez Nina Bouraoui, Algérienne de mère française, qui dit ses mixités, culturelle et sexuelle : elle est aussi lesbienne («La métaphore cognitive de la guerre chez Nina Bouraoui», pp. 245-258). Dans Garçon manqué (2000), texte issu d'une thérapie, la guerre, métaphore cognitive, structure et influence des manières de penser et d'agir duelles.

Quand on sait que, paradoxalement au vu de l'importance du phénomène, les sociologues reculent devant l'analyse de la guerre, ces réflexions, certes d'ordre littéraire, sont bienvenues. Les analyses, on le voit, s'attachent surtout aux fictions, éventuellement autobiographiques, parfois aux journaux intimes, peu aux articles, essais et correspondances de femmes (à l'exception de maigres allusions aux essais de Viviane Forrester, aux lettres de Colette et de Simone de Beauvoir), où s'est pourtant illustrée l'helléniste belge Marie Curvers-Delcourt, qui s'intègrerait parfaitement au corpus étudié. Il est vrai que la revue belge Cahiers d'histoire du temps présent -Bijdragen tot de eigentijdse geschiedenis, dans son numéro consacré au thème "Femme et société " (4, juil. 1998) par exemple, semble ignorer l'œuvre considérable de l'érudite, oubli auquel nous espérons remédier en publiant sa correspondance avec son amie la Luxembourgeoise Aline Mayrisch de Saint-Hubert. D'autres auteures belges ont écrit sur la guerre: Marie Gevers, Madeleine Bourdouxhe, Jacqueline Harpman. Bruxelles, Liège, Arlon ou Colpach, c'est aussi la francophonie, où l'on croit qu'écrire sauve.

\section{AUTEURS}

\section{CATHERINE GRAVET}

Faculté de traductionet d'interprétation -E.I.I, université Mons-Hainaut, catherine.gravet@gmail.com 